\title{
Self-Optimisation of Vertical Sectorisation in a Realistic LTE Network
}

\author{
Konstantinos Trichias*, Remco \\ Litjens ${ }^{*}, \dagger$ \\ ${ }^{*}$ TNO, ${ }^{\dagger}$ Delft University of Technology \\ Delft, The Netherlands \\ $\{$ kostas.trichias,remco.litjens\}@tno.nl
}

\author{
Abdoulaye Tall, Zwi Altman, \\ Orange Labs \\ Issy-les-Moulineaux, France \\ \{abdoulaye.tall,zwi.altman \\ \}@orange.com
}

\author{
Pradeepa Ramachandra \\ Ericsson Research \\ Linköping, Sweden \\ pradeepa.ramachandra@ericsson.com
}

\begin{abstract}
Vertical Sectorisation (VS) is a feature that exploits the capabilities of Active Antenna Systems (AAS) to improve service performance and network capacity, by splitting a single cell into an inner and outer sector. The attained gains primarily depend on the suitable and timely (de)activation of VS in response to the observed/estimated traffic distribution within the cell, while further gains can be achieved by properly adjusting VS parameters such as the transmit power distribution. Considering a realistic LTE (Long Term Evolution) network environment, in this paper a SON (Self Organizing Network) algorithm is proposed and evaluated, which dynamically (de)activates the VS feature and sets the transmit power split. The results show that the (de)activation of VS through a suitably tuned SON algorithm achieves significant throughput gains, while the dynamic adaptation of the transmit power distribution is mostly beneficial when operating in interference-limited environments.
\end{abstract}

Keywords-Vertical Sectorisation, Active Antenna Systems, SelfOrganizing Networks, realistic LTE environment.

\section{INTRODUCTION}

Mobile network operators are continuously exploring ways to increase the capacity of their LTE network in order to accommodate the mobile data traffic growth. Among various technical innovations that are proposed and investigated is the deployment of Active Antenna Systems (AAS) which promises significant capacity enhancements with a high degree of flexibility [1]. In order to fully exploit the potential of the AAS technology, complementing it with a SON functionality will ensure that the network better adapts to the spatially and temporally varying traffic demands [2].

AAS makes use of active electronic components which facilitate a large degree of freedom, thereby allowing more than one individually controlled beams from the antenna [3]. A key application of the AAS capabilities to enhance mobile network capacity is to dynamically deploy Vertical Sectorisation (VS), which refers to the use of two vertically separated beams from the same antenna.

Prior art on VS has focused on performance evaluation of VS for various sector tilts and vertical half-power beamwidths [1] and in comparison of VS with horizontal sectorisation [4]. Tsakalaki et. al [5] use a realistic AAS model and propose an enhanced vertical sector beam pattern design, supporting higher throughputs due to reduced inter-sector interference. In [6] an extensive controllability and observability $(\mathrm{C \& O})$ study of VS is carried out considering a realistic LTE network environment. Based on these results, in the current paper a
SON algorithm for the (de)activation and tuning of the VS feature is proposed and evaluated using system-level simulations, as depicted in Figure 1. The objective of this paper is twofold: $(i)$ to design a VS SON algorithm for activation/deactivation of the VS, and dynamic transmission power distribution between the inner and outer sectors; and (ii) to calibrate the algorithm and evaluate the performance of VS SON feature using a realistic network simulator.

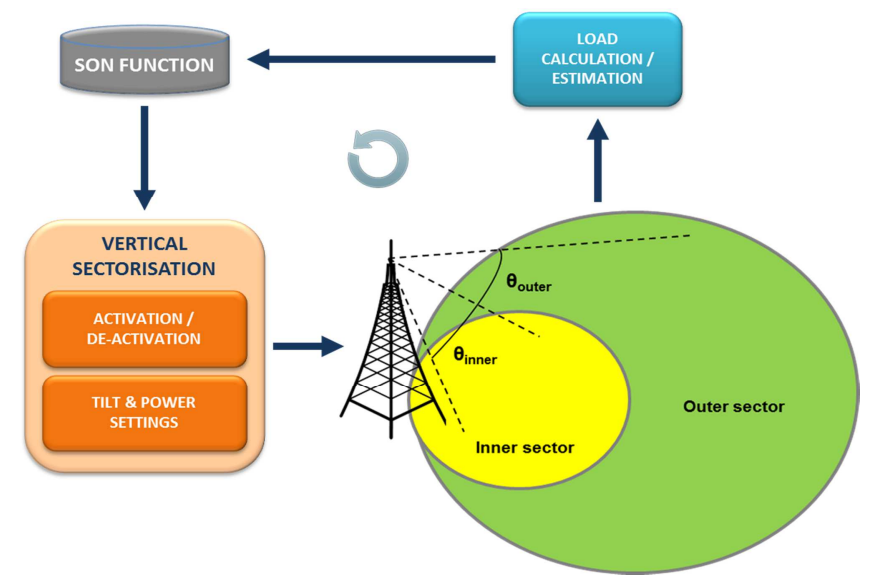

Figure 1: Vertical sectorisation operated by a SON function.

The paper is organised as follows. Modeling aspects regarding the network, traffic characteristics and propagation environment are given in Section II. Section III then specifies the proposed SON algorithm. Subsequently, Section IV outlines the assessment approach in terms of the considered scenarios. Simulation results are presented and discussed in Section V. Concluding remarks are given in Section VI.

\section{MODELING}

This section describes the key modeling aspects underlying the downlink performance assessment of the presented selfoptimisation algorithm for VS in a realistic LTE network. It is noted that the basic modelling assumptions are the same as those used in [6], and are repeated here in somewhat condensed form to ensure the paper's self-contained character.

\section{A. System Aspects}

We consider a realistic network layout of 63 outdoor LTE sites covering a $5 \times 7 \mathrm{~km}^{2}$ area in the city of Hannover, 
Germany, as depicted in Figure 2. Although the entire network area is addressed in the conducted simulations, performance statistics are collected only in the indicated central area of $3 \times$ $5 \mathrm{~km}^{2}$, covered by 36 sectorised sites.

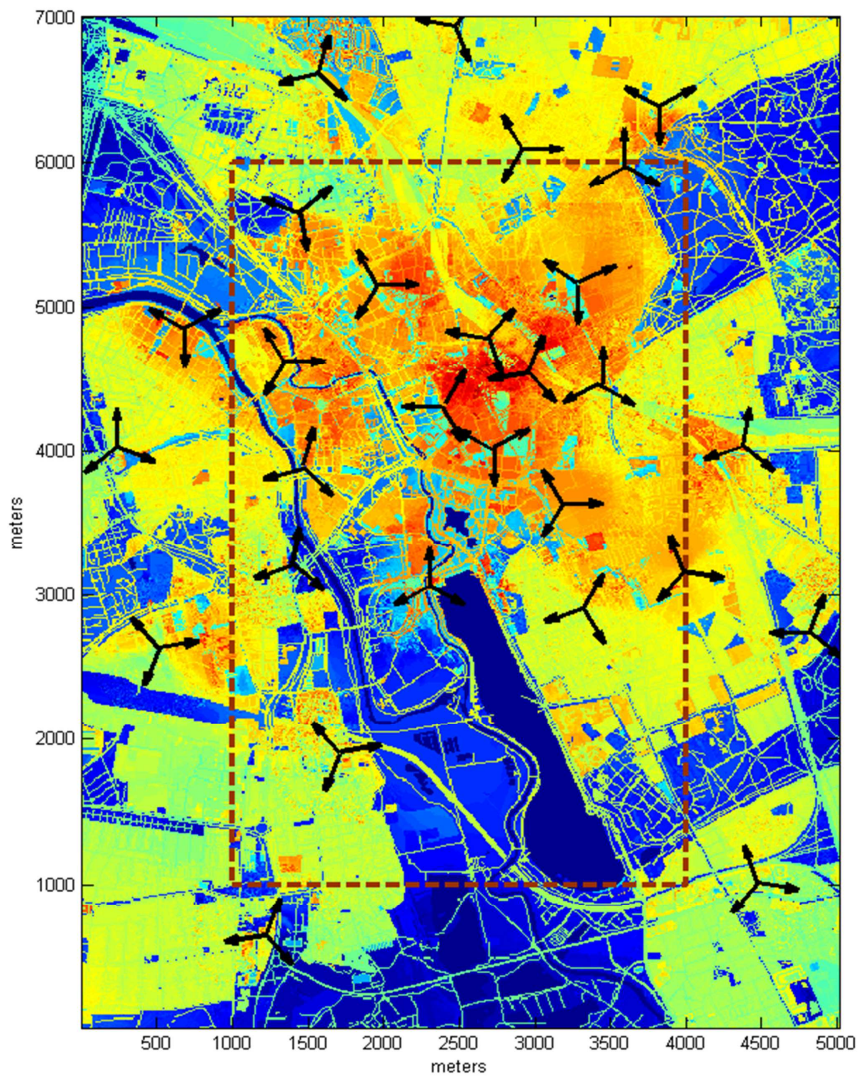

Figure 2: Considered LTE network in Hannover and spatial traffic intensity.

Each site is horizontally sectorised, as visualised in the figure, while each such cell (horizontal sector) is equipped with a 'vertically sectorisable' active antenna operating in the $1800 \mathrm{MHz}$ band with a system bandwidth of $20 \mathrm{MHz}$, a maximum transmit power $P_{\text {total }}$ of $40 \mathrm{~W}$, a main lobe antenna gain of $18 \mathrm{dBi}$ and a fixed mechanical downtilt of $4^{\circ}$. On top of this mechanical downtilt, an electrical downtilt can be applied to the unsectorised $(\theta)$ or sectorised $\left(\theta_{\text {inner }}, \theta_{\text {outer }}\right)$ antenna. The antenna model used in this work is based on [9][10]. In case of vertical sectorisation, $P_{\text {total }}$ is split into $P_{\text {inner }}$ and $P_{\text {outer }}=P_{\text {total }}-P_{\text {inner }}$, where the cell-specific power split is set by the self-optimisation algorithm described in Section III.

In the used terminology, a 'cell' refers to a horizontal sector, as depicted in theFigure 2. Vertical sectorisation of such a cell, segments it into an inner and an outer 'sector'. In the assessment of the performance impact of VS, the term 'cell' is used both for the (vertically) unsectorised service area of an antenna, and for the aggregate of the service areas of the inner and the outer sector in a sectorised setting.

\section{B. Traffic Characteristics}

Traffic is generated according to a spatially heterogeneous Poisson arrival process, with the relative traffic intensities depicted in Figure 2. This relative traffic intensity map, which is realistically based on actual measurements in the Hannover area [11], is uniformly scaled in order to obtain absolute traffic intensities. A generated session is modelled as a file download with a deterministic size of $2 \mathrm{MB}$. The area-wide average offered traffic load is expressed in $\mathrm{Mb} / \mathrm{s} / \mathrm{km}^{2}$.

\section{Propagation Environment}

Using real 3D building data and applying advanced ray tracing modelling [7], a realistic propagation model is developed to cover both in- and outdoor pixels at a pixel resolution of $10 \times 10 \mathrm{~m}^{2}$. The model covers isotropic propagation losses, comprising the effects of distance-based path loss, shadowing, diffraction, reflections and building penetration. Antenna masking is done adaptively within the simulations, depending on the specifically applied electrical down-tilts. See also[6].

\section{Resource Management}

A generated session is assigned to the cell towards which it experiences the strongest RSRP (Reference Signal Received Power), noting that the RS (Reference Signal) has the same transmit power, regardless of whether a cell is vertically sectorised or not. Given the dynamics of the number of served calls in each cell, the cell's PDSCH (Physical Downlink Shared CHannel) transmits at either full power or is idle. At any time, the experienced SINR of a given session is mapped to an attainable bit rate $R(S I N R)$, expressed in $\mathrm{Mb} / \mathrm{s}$, using the modified Shannon formula taken from [8]:

$$
R(S I N R)=B W \times \min \left\{4.4,0.6 \log _{2}(1+S I N R)\right\},
$$

with $B W$ the available bandwidth in $\mathrm{MHz}$. Assuming resourcefair channel sharing, the experienced bit rate of the considered user is then equal to $R(S I N R) / N$, with $N$ the current number of active sessions in the user's serving cell.

In the event of the sectorisation of a given cell, either the inner or the outer cell (typically the one estimated to attract the most ongoing sessions) inherits the PCI (Physical Cell ID) of the unsectorised cell, while the other cell is assigned a new PCI. Depending on the RSRP measurements, ongoing sessions may either remain attached to the former (existing) cell, or be handed over to the latter (new) cell. In an unsectorisation event, the inner cell is effecively switched off, but not before any remaining sessions are first handed over to the best alternative candidate cell. In this work, the handover process is assumed to take place instantaneously with no impact on the call performance whatsoever.

\section{SON ALGORITHM}

The fundamentals of the proposed SON algorithm which (de)activates VS based on the estimated / observed load in the inner and outer sectors of a cell has been described in [6]. A decision boundary in the inner- outer cell load plane is analytically derived, and by using data from a realistic network simulator, the functional form of the decision boundary is adjusted. The (de)activation decision is taken as a function of the position of the operation point with respect to the decision boundary [6]. In the current paper we propose a simplified version for the SON algorithm, namely we derive a decision boundary with a linear form. In Section V it is shown 
that for a reactive implementation of the SON algorithm, as soon as a mobile user enters the inner sector coverage zone, the activation of the VS is beneficial. Hence load estimation is simplified. An extension of the algorithm for dynamic adaptation of the transmit power split between the inner and outer sectors is presented as well. The SON algorithm parameters are optimised using a realistic LTE network simulator.

\section{A. (De)activation of VS}

The (de)activation of VS in a given cell (or, equivalently, the switching on/off of the inner sector), depends on the load of the inner and outer sectors, since VS is only beneficial if some part of the cell traffic is served by the inner sector [6]. The (de)activation decision is steered by the following ratio:

$$
\text { Load Ratio }=\frac{N_{\text {outer }}^{\tau}}{N_{\text {inner }}^{\tau}}
$$

where $N_{\text {inner }}^{\tau}$ and $N_{\text {outer }}^{\tau}$ are the number of data sessions served by the inner and outer sectors at each time interval $\tau$, respectively. $\tau$ also defines the timescale at which SON decisions are taken. For instance if a decision for (de)activation needs to be taken every $\tau=10$ seconds, then the Load Ratio needs to be evaluated with the same periodicity. The extreme case of $\tau=0$ seconds means that VS (de)activation decisions are made upon each session arrival / departure event.

Using the Load Ratio of each cell in a time interval $\tau$, a SON decision for (de)activation is taken every $\tau$ seconds based on a threshold $\alpha$. The parameter $\alpha$ represents the linear slope of the decision boundary used by the SON algorithm, in accordance with the following rules:

\section{if Load Ratio $\leq \alpha$ then activate VS \\ if Load Ratio $>\alpha$ then deactivate VS,}

Indeed, the lower the current Load Ratio, the higher the number of sessions that are (estimated to be) served in the inner sector, and hence the more likely that VS will be activated to accommodate them.

In order to optimize the performance of the SON algorithm, a sensitivity study was carried out in the twodimensional space of $\alpha$ and $\tau$, and the optimum values for these parameters were found. The results of this sensitivity study are shown in SectionV.

\section{B. Dynamic adaptation of the transmit power split}

Once the decision has been made to vertically sectorise a cell, the VS settings need to be defined, including the sectorspecific electrical downtilts $\left(\theta_{\text {inner }}, \theta_{\text {outer }}\right)$ and the available transmit power per sector $\left(P_{\text {inner }}, P_{\text {outer }}\right)$. The controllability study carried out in [6] indicated that for a network-wide deployment of VS in the Hannover scenario, considering static values for the VS parameters, the optimum choice is $\left(\theta_{\text {inner }}\right.$, $\left.\theta_{\text {outer }}\right)=(8,0)$ and $\left(P_{\text {inner }}, P_{\text {outer }}\right)=\left(0.5 P_{\text {total }}, 0.5 P_{\text {total }}\right)$. The latter will be referred to as the 'static power split'.

The power split between the two sectors impacts the performance of the calls in the two sectors, as well as the performance of calls in the neighbouring cells. We propose an extended 'dynamic power split' version of the SON algorithm that assigns the available transmit power to a sectorised cell according to the number of ongoing sessions in the inner and outer sectors and the number of immediately affected neighbouring sessions:

$$
P_{\text {inner }}=\frac{N_{\text {inner }}+\omega * N_{\text {interfering }}}{N_{\text {inner }}+N_{\text {outer }}+\omega * N_{\text {interfering }}} \cdot P_{\text {total }},
$$

with $P_{\text {outer }}=P_{\text {total }}-P_{\text {inner }}$. In (2), $N_{\text {interfering denotes the number }}$ of immediately affected ongoing sessions served by neighbouring cells, i.e. a session experiencing a path loss smaller than $115 \mathrm{~dB}$ to the cell in which VS is to be (de)activated, and $\omega$ is a weight ('neighbour coefficient') determining the importance of the neighbouring sessions. In this definition, the threshold value of $115 \mathrm{~dB}$ was determined numerically using extensive numerical simulations for the considered LTE network environment. Section V presents the results of a sensitivity study that allows to determine the optimum value for $\omega$.

\section{SCENARIOS}

A series of different scenarios have been simulated for the described Hannover network, in order to determine the impact of the SON function configuration parameters on the network performance and assess the performance for different load settings. This section outlines the considered scenarios and specifies the key performance metrics.

\section{A. Configuring the SON function parameters}

As described in Section III the proposed SON function is essentially characterised by three distinct parameters. The decision to (de)activate is affected by the timescale parameter $\tau$ and the threshold $\alpha$ regarding the Load Ratio [eq.(1)]. Once a cell is vertically sectorised the SON function either splits the cell power $P_{\text {total }}$ evenly over the inner and outer sector, or splits dynamically in accordance with weight parameter $\omega$.

For a plausibly heavy load scenario, characterised by an average traffic intensity of $43.8 \mathrm{Mb} / \mathrm{s} / \mathrm{km}^{2}$ (within the $3 \times 5$ $\mathrm{km}^{2}$ statistics collection area), we first tune the (de)activation part of the SON function. This is done by assessing the areawide performance for all combinations of $\alpha \in$ $\{1,2,3,4,5,10,20, \infty\}$ and $\tau \in\{0,1,5,10,20\}$. Subsequently, for the found optimal $(\alpha, \tau)$ pair, the optimal choice of the power split parameter ('static', or $\omega \in[0.0,1.0]$ ) is derived by assessing the area-wide performance for the indicated range of parameter settings and average traffic intensities of 18.7, 31.3 and $43.8 \mathrm{Mb} / \mathrm{s} / \mathrm{km}^{2}$ (low, medium and high loads).

\section{B. Assessing the gains of self-optimised vertical sectorisation}

Given the tuned configuration of the self-optimisation algorithm, in the second part of the assessment we determine the network performance for the cases of ' $N o V S$ ' (no cells are vertically sectorised), 'Full $V S$ ' (all cells are vertically sectorised with an electrical downtilt of $8^{0}$ for the inner sector and a power split of $50 \%$ - 50\%, see [6]) and 'SON VS' (vertical sectorisation is dynamically (de)activated by the tuned SON function), and for a range of traffic loads. The 
performance is assessed based on network-wide Key Performance Indicators (KPIs) as well as local-area KPIs, in order to learn whether the gains from vertical sectorisation depend e.g. on specific cell characteristics. Specifically, we will investigate to what extent the gains depend on the intersite distances (ISD), by classifying cells accordingly.

\section{Performance metrics}

The reported performance metrics concentrate on the user throughput. For an individual session, the attained user throughput is equal to the file size (2 MB) divided by the session duration. Based on the sessions terminated after an adequate warm-up time, the network-wide average and $10^{\text {th }}$ percentile of the user throughput are determined. Furthermore, the average and $10^{\text {th }}$ percentile of the user throughput are determined on a per-cell basis, recalling from the terminology introduction in Section II.A that 'cell' has a well-defined meaning in both a sectorised and an un-sectorised setting.

\section{NUMERICAL RESULTS}

This section presents the performance results for the SON algorithm proposed in Section III and implemented in the realistic network simulator described Section II. We begin by assessing the optimal parameters to be used by the VS SON algorithm when a static power split is used (50\%-50\%). We present the performance obtained with varying values of $\alpha$ (the slope of the linear controller) and $\tau$ representing the frequency of updates of the SON algorithm.

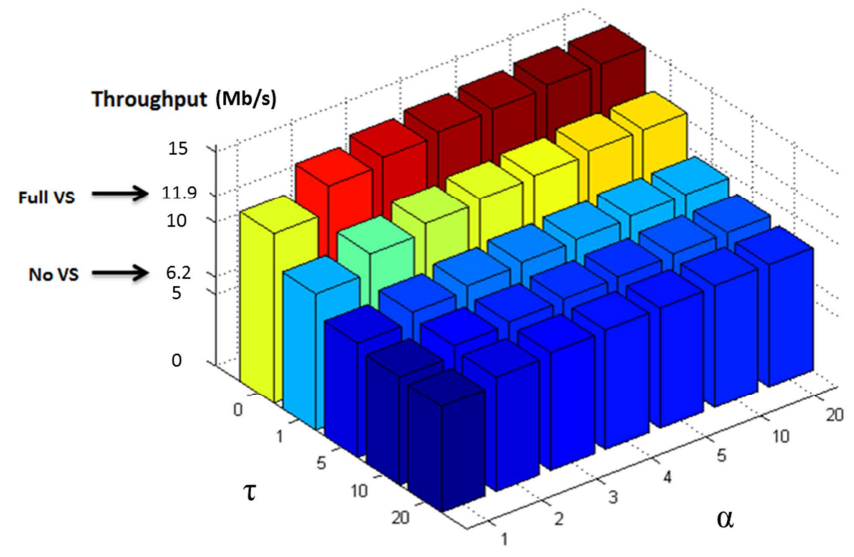

Figure 3: $10^{\text {th }}$ user throughput percentile versus the SON load ratio threshold $\alpha$ and the SON decision timing parameter $\tau$.

Figure 3 shows that the best slope $\alpha$ for the SON algorithm tends to infinity. Indeed, the performance increases with $\alpha$ and converges for $\alpha \geq 10$. This implies that it is beneficial to activate the VS feature when there is at least one session in the inner sector. As for $\tau$ the best value is 0 , meaning that VS (de)activation decisions should be made upon each session arrival / departure event. In the following, the VS SON parameters of $\tau=0$ and $\alpha=\infty$ are adopted.

Next, we assess the effect of having a SON algorithm with a dynamic power split (considering different neighbour coefficients $\omega$ ) over having a static power split, for a high load scenario with an average traffic demand of $43.8 \mathrm{Mb} / \mathrm{s} / \mathrm{km}^{2}$. Initial results showed that the difference in performance between the static and dynamic power split is negligible when network-wide KPIs are considered. As the dynamic power split is expected to specifically offer advantages when applied in interference-limited environments, we concentrate on the most densely deployed urban environment. Figure 4 shows the $10^{\text {th }}$ user throughput percentile, for different values of $\omega$, for cells with an average Inter-Site Distance (ISD) to their ten closest neighbours of less than 500 meters.

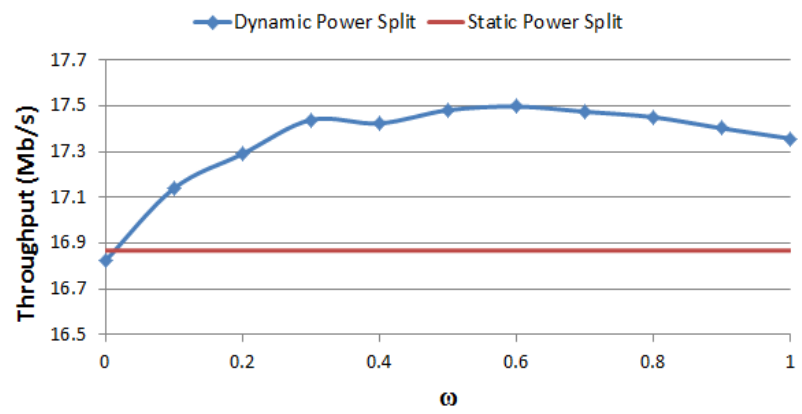

Figure 4: $10^{\text {th }}$ user throughput percentile for static and dynamic power split SON as a function of the neighbour coefficient $\omega$, for cells with ISD $<500 \mathrm{~m}$.

From Figure 4 it can be observed that the dynamic power split can offer an improvement of up to $4 \%$ in $10^{\text {th }}$ user throughput percentile, considering interference-limited environments. An improvement of up to $5 \%$ is observed for the average user throughput. The gains observed are rather small due to the trade-off between serving some users with higher power and creating stronger interference for some other users. Even so, the performance of the dynamic power split constitutes an improvement over the static power split and hence we conclude that the optimal case is to have a dynamic power split with a neighbour coefficient of $\omega=0.6$.

In the remainder of this section, we compare the performance of the optimised dynamic power split SON algorithm denoted ' $S O N V S$ ' with the reference cases denoted 'No VS' and 'Full VS', as described in Section IV.

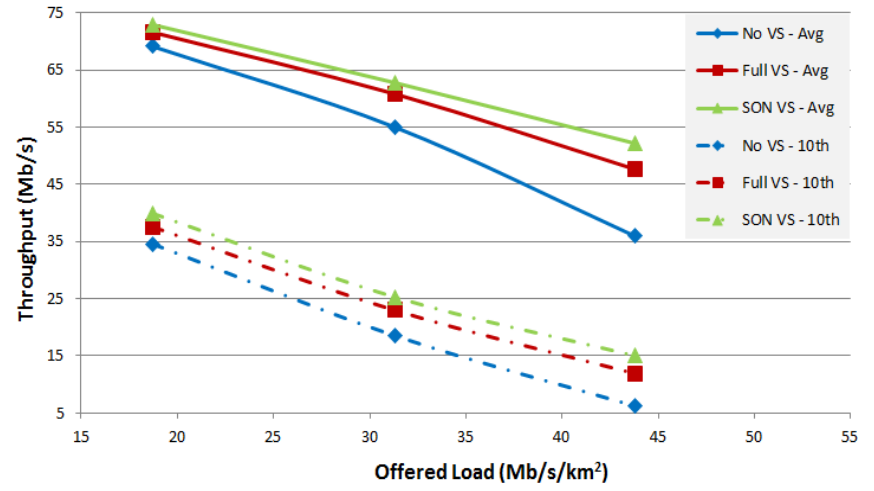

Figure 5: Average and $10^{\text {th }}$ user throughput percentile versus the offered traffic load 
Figure 5 present the average and $10^{\text {th }}$ user throughput percentiles for the entire network, for a range of offered traffic loads. This figure shows the gain achieved by the VS SON algorithm which increases with the load and can reach $45 \%$ for average user throughput and $140 \%$ for the $10^{\text {th }}$ user throughput percentile, compared to the ' $N o V S$ ' case, for the heaviest load simulated. The corresponding throughput gains of the 'SON VS' scenario compared to the 'Full VS' case are $9.5 \%$ and $26 \%$, respectively.

Network-wide KPIs such as the ones used in Figure 5 only give an overview of the performance. In order to understand how SON VS affects the different cells, local area KPIs need to be used. Figure 6 and Figure 7 depict the average user throughput and the $10^{\text {th }}$ user throughput percentile, respectively, for cells grouped according to their average ISD (to their ten closest neighbours) for the highest offered load. It is noted that the Hannover area includes different environment types, i.e. dense urban, urban and suburban.

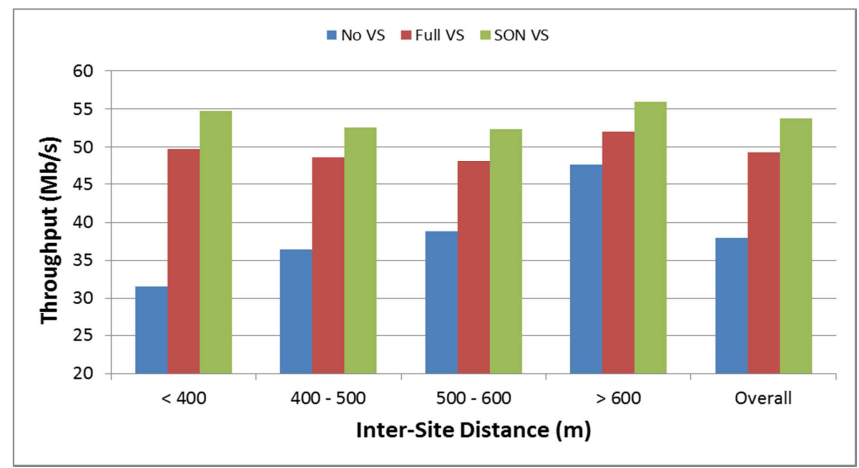

Figure $6:$ Average user throughput for cells with different ISDs.

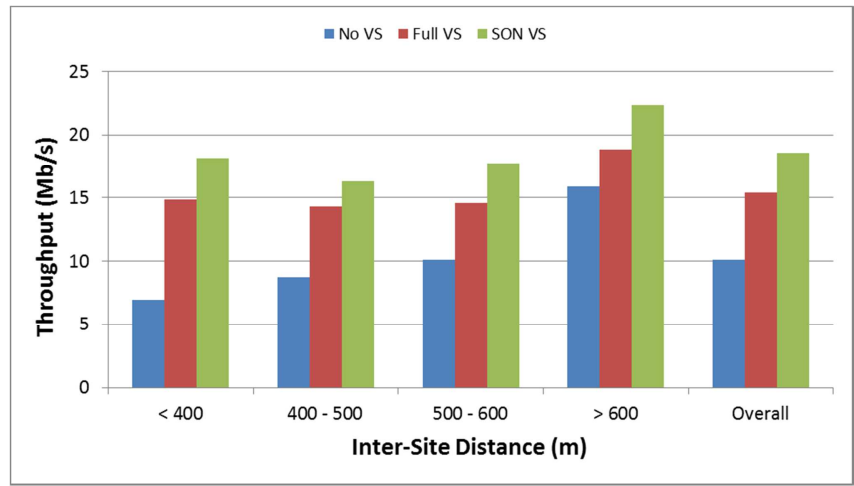

Figure 7: $10^{\text {th }}$ user throughput percentile for cells with different ISDs.

One can see from Figure 6 and Figure 7 that the throughput gain achieved by the VS SON algorithm, is more significant for cells with smaller ISDs (urban cells). In fact, the larger the average ISD of a cell the smaller the gains it experiences from deployment of VS, with a SON algorithm or without. This result is mainly due to the higher traffic load in the urban areas, that can be better served by the additional capacity provided by VS. This additional capacity enables the network to serve data traffic with higher throughputs, consequently reduce session download times and hence also the associated interference that is caused.

\section{CONCLUDING REMARKS}

This paper has presented a novel SON algorithm for the dynamic activation and tuning of the VS feature, along with simulations results of this SON algorithm obtained from a system-level simulator for a realistic LTE network environment. The proposed SON algorithm is shown to perform best when the controller decisions are taken upon session arrival / departure events and it can lead to an increase of up to $26 \%$ in $10^{\text {th }}$ user throughput percentile compared to a VS deployment without SON. The dynamic adaptation of transmit power to traffic distribution has been shown to only offer gains of up to $5 \%$. Future work will focus on dynamic tilting of the inner sector as well as a first assesment of the performance of Virtual Sectorisation (ViS).

\section{Acknowledgment}

The research leading to these results has been carried out within the FP7 SEMAFOUR project and has received funding from the European Union Seventh Framework Program (FP7/2007-2013) under grant agreement no 316384.

\section{References}

[1] O. Yilmaz, S. Hamalainen, J. Hamalainen, 'Self-Optimization of Coverage and Capacity in LTE using Adaptive Antenna Systems', Master's thesis report, Feb 2010.

[2] J. Ramiro and K. Hamied, Self-Organizing Networks (SON): SelfPlanning, Self-Optimization and Self-Healing for GSM, UMTS and LTE. John Wiley \& Sons Ltd, 2011.

[3] 3GPP TR 37.840, 'Technical Specification Group Radio Access Network Study of AAS Base Station (Release 11)'

[4] M.Caretti, M.Crozzoli G.M Dell'Aera, A.Orlando "Cell splitting based on Active Antennas: Performance Assesment for LTE System", Telecom Italia, 2012.

[5] Elpiniki P. Tsakalaki, Luis Angel Maestro Ruiz de Temino, Tomi Haapala, Javier Lopez Roman and Miguel Arranz Arauzo, "Deterministic Beamforming for Enhanced Vertical Sectorisation and Array Pattern Copensation", Aalborg University, Nokia-Siemens Netwroks, Vodafone Technology Networks, 2011.

[6] K.Trichias, R.Litjens, A.Tall, Z.Altman, P. Ramachandra, 'Performance evaluation \& SON aspects of vertical sectorisation in a realistic LTE network environment', $11^{\text {th }}$ International Symposium on Wireless Communications Systems (ISWCS), 2014.

[7] Y. Lostanlen and T. Kürner, 'Ray-Tracing Modeling' in 'LTE Advanced and Next Generation Wireless Networks: Channel Modeling and Propagation', Wiley, 2012.

[8] 3GPP, 'Evolved Universal Terrestrial Radio Access (E-UTRA); Radio Frequency (RF) system scenarios', TR 36.942, v11.0.0, 2012.

[9] 3GPP TSG-RAN WG4 meeting \#62, R4-120908, "Proposal to modeling active antennas", Kathrein, Feb. 2012.

[10] 3GPP TSG-RAN WG4 meeting \#62bis, R4-121835, "Modeling Active Antennas", Katherein, Mar, 2012.

[11] D.M. Rose, T. Jansen, T. Werthmann, U. Türke and T. Kürner, 'The IC 1004 Urban Hannover Scenario - 3D Pathloss Predictions and Realistic Traffic and Mobility Patterns', COST IC1004 TD(13)08054, Ghent, Belgium, 2013. 